
\title{
25 Research Soure \\ The features of polyglutamine regions depend on their evolutionary stability
}

Pablo Mier ( $\square$ munoz@uni-mainz.de)

Johannes Gutenberg University Mainz https://orcid.org/0000-0003-3663-2352

Miguel A. Andrade-Navarro

Johannes Gutenberg Universitat Mainz

Research article

Keywords: Homorepeat, polyQ, glutamine, codon usage, evolutionary stability

Posted Date: February 28th, 2020

DOI: https://doi.org/10.21203/rs.3.rs-15440/v1

License: (9) (1) This work is licensed under a Creative Commons Attribution 4.0 International License. Read Full License

Version of Record: A version of this preprint was published at BMC Evolutionary Biology on May 24th, 2020. See the published version at https://doi.org/10.1186/s12862-020-01626-3. 


\section{Abstract}

Background Polyglutamine regions (polyQ) are one of the most studied and prevalent homorepeats in eukaryotes. They have a particular length-dependent codon usage, which relates to a characteristic CAGslippage mechanism. Pathologically expanded tracts of polyQ are known to form aggregates and are involved in the development of several human neurodegenerative diseases. The non-pathogenic function of polyQ is to mediate protein-protein interactions via a coiled-coil pairing with an interactor. They are usually located in a helical context.

Results Here we show how these known features related to polyQ depend on their stability in evolution. We have classified the polyQ regions of 60 proteomes from four distinct taxonomic groups (Insecta, Teleostei, Sauria and Mammalia) in three main categories based on their evolutionary stability. Codon usage, amino acid context, structural conformation and the protein-protein interaction capacity of polyQ from all studied taxa critically depend on the region stability.

Conclusions Our results show that apart from the sequence of a polyQ, information about its orthologous sequences is needed to assess its function.

\section{Background}

Polyglutamine regions (polyQ) are homorepeats defined by a higher local proportion of glutamines than expected in protein sequences. Due to this relaxed definition, multiple combinations of glutamines and non-glutamine residues (impurities) may form a polyQ. A minimum of four glutamines in a six-amino acid region are nonetheless required (Totzeck et al., 2017).

PolyQ are undoubtedly the most studied homorepeat for various reasons. First of all, it is one of the most prevalent homorepeats in eukaryotic proteomes (Faux et al, 2005; Jorda and Kajava, 2010; Lobanov and Galzitskaya, 2012; Mier et al., 2017). Second, from the point of view of the nucleotide composition of the corresponding coding region, it is a relatively simple homorepeat to study, as glutamine is coded by only two codons, CAG and CAA. Third, they are associated to ten neurodegenerative diseases (Blum et al., 2013), elicited by CAG expansions. And last, it is one of the few homorepeats for which an expansion mechanism has been proposed, a replication-mediated slippage (Albà et al., 2011).

As a result, much is known about these homorepeats. Almost non-existent in archaea and bacteria, they are widely present in eukaryotic species (Mier et al., 2017; Lobanov et al., 2014). Codon usage is speciesand polyQ-length dependent, but they are usually highly enriched in CAG over CAA; as an example, in mammals they are coded by a 3:1 proportion of CAG:CAA (Mier and Andrade-Navarro, 2018). They can be found in multiple lengths and with variable quantities of impurities within (Mier et al., 2020). The association between polyQ length and impurity distribution is highly species-dependent, but generally shorter pure polyQ prevail. Regarding impurities, leucine and proline residues are common in impure polyQ (Mier et al., 2020). Both these residues also play a role in the amino acid context of some polyQ regions. The former is enriched in position - 1 of polyQ in vertebrates (Mier et al., 2020); it has been 
proposed that it promotes an alpha-helical structure towards the polyQ, making it less aggregation prone (Eftekharzadeh et al., 2016; Escobedo et al., 2019). The latter follows long human polyQ in the form of a proline-rich or polyP region (Schaefer et al., 2012), also to limit the aggregation propensity of the long polyQ (Bhattacharyya et al., 2006; Darnell et al., 2007). These sequence mechanisms surrounding polyQ regions are in place to attenuate a polyQ length-dependent aggregation process, which in humans ultimately leads to disease (Yushchenko et al., 2018). Protein aggregation in this case is a byproduct of an abnormally long polyQ that has to do with its wild-type biological role in stabilizing protein-protein interactions (Schaefer et al., 2012); new abnormal interactions may be gained by the extended polyQ resulting in aggregates. Regarding the structural conformation of polyQ regions, it has been described that they are preceded by a helical conformation and followed by random coil (Totzeck et al., 2017).

It has been theorized that there are two possible ways for a polyQ region to emerge in a sequence: by replication slippage and by point mutations (Albà et al., 2001). Albà and colleagues described the different codon usage of polyQ regions from seven model organisms based on these mechanisms. Whether any other polyQ feature is influenced by its emergence mechanism is yet to be described. To the best of our knowledge, no other study since then has tried to take these mechanisms into account when studying polyQ regions or any other homorepeat. Our aim is to challenge the established knowledge about polyQ features and to classify polyQ based on the way they emerged (or are emerging) in a sequence. While they use the nucleotide sequence to classify the regions, our take is to classify them based on the amino acid sequence, and to establish whether a polyQ is stable or unstable in evolution and how the degree of stability relates to their amino acid sequence and function.

Here we use the proteome of 60 species from four different taxa (Class Insecta, Infraclass Teleostei, Clade Sauria and Class Mammalia) to generate sets of orthologs of polyQ-containing proteins. Then we classify the orthologous polyQ regions in three categories based on their stability, that is, general conservation of the glutamine residues. Nearly all of the polyQ features described above are studied based on these three polyQ stability categories. We show how all known features are category-dependent, and thus evolutionary information is needed to establish general rules as to the behavior of polyQ regions.

\section{Results}

\subsection{PolyQ regions can be categorized based on their evolutionary stability}

A protein sequence is thought to be something static, a fixed ordered set of amino acids reflecting the biological entity that is a protein. But in reality, the same sequence is constantly under selective pressure that may be favoring sequence alterations. Here we aim to study whether polyQ regions can be categorized based on their evolutionary stability. We define this stability comparing polyQ regions across orthologs from taxonomically close species.

We prepared aligned sets of orthologs in which at least one protein has a polyQ region (see Methods for details), for four distinct taxonomic groups: Class Insecta, Infraclass Teleostei, Clade Sauria and Class 
Mammalia. The aligned polyQ regions were then studied in detail to assess the glutamine conservation per aligned position (Figure 1). Each position has three possible values, depending on the proportion of sequences having a glutamine:
a. conserved, if $>=80 \%$ of the sequences have a glutamine;
b. unstable, if $<80 \%$ but $>=20 \%$ of the sequences have a glutamine; and
c. uncategorized, if $<20 \%$ of the sequences have a glutamine.

For (a) and (c) it is clear that the glutamine in that position is clearly conserved or absent in the alignment, respectively. In the case of (b), we further defined three sub-values:

1. inserted, if there are more gaps than non-glutamine amino acids;

2. mutated, if there are more non-glutamine amino acids than gaps;

3. undefined, if the number of gaps and non-glutamine amino acids is the same.

The (b) value reflects an aligned position not dominated by glutamines, being the sub-value an indicator of the possible underlying mechanism driving the position. More gaps would mean a length variation in the sequence, whereas any residue different to glutamine would imply a point mutation either to or from the glutamine.

The stability of the polyQ is categorized by taking into consideration all the values of glutamine conservation along the complete span of the polyQ region. Five categories are defined:

- Category 1, stable polyQ. More (a) conserved than (b) unstable positions.

- Category 2, unstable polyQ by length variation. More (b) unstable than (a) conserved positions, and more (b1) inserted than (b2) mutated and (b3) undefined.

- Category 3, unstable polyQ by mutations. More (b) unstable than (a) conserved positions, and more (b2) mutated than (b1) inserted and (b3) undefined.

- Category 4, unstable polyQ by undefined mechanism. More (b) unstable than (a) conserved positions, and not in category 2 or 3 .

- Category 5, uncategorized. Not in any previous category.

Although five categories are defined, only in 1,2 and 3 it seems clear how the stability or instability of the polyQ region is achieved in the orthologs. In category 4 it is theoretically not possible to determine whether the instability comes from an indel or a point mutation, and category 5 is by definition place for the uncategorized regions. For the purpose of the present work we discard hereafter the polyQ regions in categories 4 and 5, and refer to the selected categories as stable (1), inserted (2) and mutated (3).

Stating that a polyQ is stable means that it is conserved in at least $80 \%$ of the orthologs. It is implied that the categorization of the polyQ is intrinsically affected by the selection of the species to use in the study (Figure 2a), as more similar orthologs would mean more stable polyQ. However, this relation is not 
straightforward, and the sequence similarity in the orthologs (Figure $\mathbf{2 b}$ ) is not directly correlated with having more stable polyQ regions (Figure 2c). Nonetheless, with our choice of species the number of polyQ regions per taxa is high enough for our purposes of obtaining statistically significant results (2639, 3588, 1884, 2639 in Insecta, Teleostei, Sauria and Mammalia, respectively). For the record, the number of discarded polyQ (categories 4 and 5) was of 1496, 740, 359, and 388 in Insecta, Teleostei, Sauria and Mammalia, respectively.

\subsection{Glutamine codon usage depends on polyQ stability category}

Glutamine codon usage is biased towards CAG over CAA codons, especially in polyQ regions of Chordata species (3:1 proportion) (Mier and Andrade-Navarro, 2018). Here we intend to determine whether there is a difference in this behavior based on the stability category of the polyQ region. To this end, we studied the codon usage in all glutamines within the orthologous polyQ regions. This means that not all considered glutamines are necessarily part of a polyQ, but they are in at least one of the orthologs. Even if they are not forming a polyQ themselves, placing them in an evolutionary context by studying their orthologs could show whether they have sequence or structural features in consonance with forming a polyQ.

PolyQ regions in the chordate taxa (Teleostei, Sauria and Mammalia) are significantly enriched in CAG codons, close or slightly above the background codon ratio CAG to CAA of 3:1, as expected (Figure 3b-d). Differently, glutamine codon usage in polyQ from Insecta does not differ much from their background, which is close to 1:1 (Figure 3a); an unexpected decrease in the \%CAG is even apparent in stable polyQ. According to the values of significance comparing the distributions, the most significant differences are higher \%CAG in inserted polyQ in Insecta and Mammalia, and different \%CAG in stable polyQ in Teleostei (higher) and in Sauria (lower). Separation above the background is higher in Sauria and Mammalia than in Insecta and Teleostei, with highest \%CAG in inserted polyQ. This is in line with previous results showing that trinucleotide repeats are subject to a CAG-slippage mechanism (McMurray, 2010).

The categories of polyQ stability can be significantly distinguished by their \%CAG, but the differences depend on the taxa. Results suggest that the CAG-slippage mechanism for which polyQ regions vary in length are predominant in inserted polyQ in Amniota species (comprising taxa Sauria and Mammalia).

\subsection{The polyQ amino acid context is influenced by polyQ stability}

Next, we study the sequences surrounding the polyQ regions. In this respect, previous work have described how polyQ regions are usually followed by a proline-rich or polyP region (Schaefer et al., 2012). In addition, we recently described an unusual peak of leucine residues in position - 1 of polyQ regions from several taxonomically diverse species (Mier et al., 2020). Here, we took one sequence at random from each set of orthologous regions, but forcing the selected sequence to have a polyQ. We then calculated the proportion of both leucine and proline residues in the previous (from position -10 to -1) and following (from position +1 to +10 ) regions. 
Although the results do not seem to be steady for leucine residues in any of the taxa, the highest proportion in position - 1 is always achieved in stable polyQ (Figure 4a). This is especially clear for mammalian sequences. It must be noted that it is difficult to draw conclusions from the noisy results from Insecta sequences due to the low amount of sequences considered (123 stable polyQ, versus, for example, 1761 stable polyQ in Mammalia). We also note that the threshold used in this work to look for polyQ regions, $4 / 6$, results in a lower signal for leucines in position -1 than other thresholds $(4 / 4,6 / 6,6 / 8$, $8 / 10$ ) when examining human proteins (Mier et al., 2020). While the $4 / 6$ threshold maximizes the number of polyQ found, features specific to longer polyQ may be obscured by the larger number of short polyQ.

Almost the complete $\mathrm{N}$-terminal and $\mathrm{C}$-terminal surrounding sequences of inserted poly $\mathrm{Q}$ regions in the four taxa are enriched in prolines, specially C-terminally to polyQ in mammalian sequences (Figure $4 \mathrm{~b}$ ). The signal is also present to some extent in mutated polyQ. The C-terminal capping of polyQ regions has been proposed as a protective mechanism against the aggregation they induce (Bhattacharyya et al., 2006). As aggregation propensity is polyQ-length dependent (Yushchenko et al., 2018), it is not surprising that inserted polyQ (which is defined by having varying length) is the category clearly showing this position-specific enrichment. We observed a remarkable decay in proline frequency in position -2 , shared by all categories in all taxa. Prolines in that position would difficult the function of polyQ in expanding a preceding alpha-helix upon protein interaction, since prolines act as helix breakers.

\subsection{Stable polyQ have higher tendency to be preceded by helical structure}

We previously reported that polyQ regions have a tendency to be preceded by a sequence in helical conformation and followed by a region in random coil conformation (Totzeck et al., 2017). Following the strategy of previous sections, and assuming that the secondary structure of all orthologous sequences will be similar, we predicted the secondary structure of one protein per set of orthologs using the tool JPred (Cuff and Barton, 1999). More specifically, we used as input sequence the polyQ region plus its sequence context. JPred uses a neural network to classify each residue to be structured as alpha helix (helical), beta sheet (extended) or as other secondary structure.

In all polyQ categories and taxa there is higher helical conformation content N-terminally to the polyQ (with a steep increase from the N-terminal side for stable polyQ; Figure 5). The aggregation of structural predictions by taxa (last column) and by category (bottom row) highlights that the category in which a polyQ is classified (stable, inserted or mutated) is more relevant than the taxa with respect to its surrounding secondary structure.

Using the results given by JPred we also studied the solvent accessibility of the residues in the input sequences. However, we found no differences between taxa or categories (data not shown). Similarly, we predicted the coiled coil propensity of the sequences using DeepCoil and also found no significant differences (data not shown).

\subsection{The protein-protein interaction capacity of a polyQ is affected by its stability}


PolyQ regions are associated to protein-protein interactions (PPI) (Schaefer et al., 2012). Propensity of a polyQ region to interact may be related to its stability category, as the structural differences described in the previous section may play a role in its interaction capacity. We considered all proteins from the sets of orthologs, independently of whether they or their orthologs have the polyQ region, and calculated the number of high confidence interactors per protein described in the STRING database. We discarded the proteins for which STRING had no entry as we could not distinguish whether they have indeed no interactors or the protein is missing in the database.

Results show that stable polyQ are present in proteins that have significantly and consistently in all taxa more interactors than proteins with unstable polyQ (Figure 6a-d). As longer proteins tend to have more interactions (Schaefer et al., 2012), we calculated the protein length distribution of the proteins to rule out the possibility that our results would be due to stable polyQ being predominant in longer proteins: this is not the case (Figure $6 \mathrm{e}$-h). Stable polyQ do then have greater number of interactors than unstable polyQ and this is not influenced by the protein length. However, proteins with many interactors are more conserved and this could explain the results.

\subsection{Functional differences of polyQ categories}

We have studied so far how the stability of a polyQ region affect its features on the nucleotide (codon usage), amino acid (sequence context), structural (secondary structure) and interaction (PPI) level. Lastly, we aim to check whether the polyQ functionality is dependent on its stability. For this purpose, we performed GO enrichment analyses of all proteins from the sets of orthologs for a representative species per taxa. Analyses were done individually per category and species.

To ease the interpretation of results, we extracted the top-10 enriched GO terms per dataset and analyzed the commonalities between categories within each species (Figure 7). Not surprisingly, the term 'coiled coil' is the top enriched term in almost all studied conditions since polyQ are known to mediate coiled-coil interactions (Schaefer et al., 2012). More interesting is the enrichment of the term 'triplet repeat expansion' exclusively for inserted polyQ of human proteins, pointing towards the correct classification of proteins depending on the polyQ categories. Regarding subcellular location, while stable polyQ are enriched in cytoplasm and nucleus, inserted polyQ are enriched only in nucleus, and mutated polyQ in cilium.

Analyses were performed independently per species, with sets of proteins specific per taxa, and as such results should not necessarily be similar between species. However, it is interesting to see how some of them are comparable. As an example, GO terms related to 'transcription' are mostly limited to inserted polyQ. On the other hand, 'cilium' and 'axon' related terms are enriched only in mutated polyQ; both cellular structures are known to share proteins (Guemez-Gamboa et al., 2014).

We illustrate the presence of mutated polyQ in human proteins annotated with the term 'cilium' with the OFD1 protein (UniProtKB ID: 075665) encoded by the Ofd1 gene (Oral-facial-digital syndrome 1). This protein is required for the formation of primary cilia (Ferrante et al., 2006). It is located in the distal 
centriole, at the cilium-centriole interface, and controls centriole length (Singla et al., 2010). To examine the structural context of the mutated polyQ regions in this protein and the other proteins annotated with the GO term 'cilium' (20 proteins in total), we searched for solved structures of the proteins or their homologs (using the web tool Aquaria (O'Donoghue et al., 2015)). Unfortunately, there was no information for or near the polyQ regions. For OFD1 we created a multiple sequence alignment including homologous and orthologous proteins of the human OFD1 chosen from selected organisms, assisted by ProteinPathTracker (Mier et al., 2018) (Figure 8a). The polyQ region ('QQEQDQ', amino acid positions 965971 ) is aligned in a block with gaps but with variable $Q$ content. The position is very close to the Cterminal of a coiled region (Figure $\mathbf{8 b}$ ), which is consistent with the function expected for polyQ.

\section{Discussion}

We have shown that there is a relation between polyQ stability and their sequence and structural context. The stability state of a polyQ region is here defined as the divergence of the sequence compared to close orthologs. A stable 'fixed in evolution' polyQ may mean that there are sequence or structure constraints that prevent it to vary in length or mutating. While this definition of stability depends on the orthologs used for comparison, in our study we appreciated sequence and structure context signals in stable polyQ that do not depend on the conservation of the orthologous set used.

Our results suggest that while there are differences between the taxa considered, stable polyQ would be generally the one more associated to a strong helical signal, both in sequence context (largest frequency of leucine at -1 position and depletion of proline at -2 position; Fig. 4) and structure context (C-terminal steep gradient of helical content; Fig. 5).

We have tried to associate global protein properties (number of protein interactors, and function) to the presence of a stable, inserted or mutated polyQ, which is a local feature. We are aware that this comparison is intrinsically problematic because for example, a protein with many interactions could be more constrained in evolution, resulting in having more stable polyQ (Fig. 6). Similarly, the heterogeneity observed in the functional terms enriched in proteins with inserted or mutated polyQ could be also influenced by different evolutionary restrictions affecting proteins with particular functions (Fig. 7).

It is reasonable to expect that longer proteins will be able to accommodate insertions and deletions more easily. This is consistent with our finding that inserted polyQ are more frequent in longer proteins (Fig. 6eh). Then, given shorter proteins, those interacting with multiple proteins competing for the same interface (for example, nuclear protein transporters that recognize multiple cargo proteins) will not be able to modify their interacting interface because it would require modifications in all proteins using it. Differently, shorter proteins using specific interfaces may be able to accommodate a polyQ even if structurally constrained to fit some length requirement because mutations can be compensated in the single target protein specifically using the interface. This would explain why stable polyQ is more frequent in proteins with more interactions than those with mutated polyQ. 
We have progressed in the last few years in the knowledge of several aspects of polyQ regions (Schaefer et al., 2013; Totzeck et al., 2017; Mier and Andrade-Navarro, 2018; Mier et al., 2020). Here we have shown that, however, there is still much to study about them. To the best of our knowledge this is the first attempt in categorizing any type of homorepeat region based on sequence data but critically depending on an evolutionary perspective. Identical homorepeat sequences may have different sequence features, because, at least for polyQ regions, it is not just the simple concatenation of glutamine residues the important factor but the stability of the region in which it is placed. The question remains as to whether this is a distinct behavior of polyQ regions or a general feature of all homorepeats.

\section{Methods}

\subsection{Data retrieval}

Protein and CDS sequences were downloaded from the FTP sites of Ensembl release-97 and Ensembl Metazoa release-44 (Zerbino et al., 2018) for a set of 60 species (Suppl. File 1).

Taxonomic information for these species was downloaded from the NCBI Taxonomy resource Common Taxonomy Tree (Sayers et al., 2009). Taxonomic trees were built using the $\mathrm{R}$ library phyloseq version 1.29.0 (McMurdie and Holmes, 2013).

Protein-protein interaction information was downloaded from the database STRING version 11.0 (Szklarczyk et al., 2019). Information was parsed so that we only take into account high-confidence interactions (score >0.7).

\subsection{Generation of sets of orthologous polyQ regions}

We took all protein sequences per proteome and performed Reciprocal Best Hits BLAST (RBHB) searches (Ward and Moreno-Hagelsieb, 2014) with an in-house script and default parameters against the rest of the proteomes in the same taxa, one at a time, to find an orthologous sequence in the maximum number of proteomes. At least five species must have an ortholog to keep the orthologous set. To avoid redundancy, a protein was allowed to be in only one orthologous set. Orthologous sets were then aligned with MAFFT v7.310 with default parameters (Katoh and Standley, 2013).

The alignments of orthologous sequences were scanned to look for polyQ regions, using a 4/6 threshold (a minimum of four glutamines in a window of six amino acids). This threshold was selected to detect the maximum number of polyQ regions following previous work (Totzeck et al., 2017; Mier et al., 2020). Orthologous regions in which at least one sequence had a polyQ were extracted from the alignments and annotated as orthologous polyQ regions. They were studied independently, making it possible to have more than one of these regions per alignment.

Pairwise-distance matrices were calculated by ClustalOmega v.1.2.4 (Sievers et al., 2011) with the alignments of the orthologous sets generated by MAFFT. An average percentage of identity per alignment was calculated for each set of orthologs. 


\subsection{Secondary structure prediction}

Assuming that the secondary structure of a protein is the same in all orthologs, we take one sequence at random per aligned polyQ and extract the region comprising the twenty previous amino acids $(-20$ to -1$)$, the polyQ, and the twenty following amino acids $(+1$ to +20$)$. We obtained 13704 sequences following this procedure (4121 in Insecta, 4321 in Teleostei, 2238 in Sauria and 3024 in Mammalia). The secondary structure and the solvent accessibility of these sequences were predicted using a schedule mass submission in JPred RESTful API v.1.5 (Drozdetskiy et al., 2015). Results are plotted from position -10 to position +10 to avoid border effects in the secondary structure sequence predictions.

For the same set of sequences we predicted their coiled coil propensity using DeepCoil (Ludwiczak et al., 2019), with a cutoff of $>=0.5$ per residue.

\subsection{GO enrichment analysis}

One model organism per taxa was selected to perform the GO enrichment analyses: Drosophila melanogaster, Danio rerio, Gallus gallus and Homo sapiens. All proteins from the set of orthologs from these four species were retrieved, and their Ensembl protein ID were mapped to either UniProt accession numbers using the ID mapping resource provided by UniProt (Huang et al., 2011) (D. rerio, G. gallus and $H$. sapiens) or to FlyBase gene ID using the Convert ID tool provided by the FlyBase database version FB2019_04 (Thurmond et al., 2019) (D. melanogaster). The mapped IDs were distributed in independent files depending on the category of the polyQ they contain (or at least one of their orthologs). These ID lists were used to perform the GO enrichment analyses in DAVID v6.8 (Huang et al., 2009) per organism and per category. Number of IDs successfully mapped to DAVID IDs per polyQ category $(1,2,3)$ were: 115 , 785 , and 1267 for D. melanogaster, 978,562 , and 890 for D. rerio; 562, 137, and 163 for G. gallus; and 1369,264 , and 488 for $H$. sapiens. Selected annotations were UP_KEYWORDS, GOTERM_BP_DIRECT, GOTERM_CC_DIRECT and GOTERM_MF_DIRECT. Duplicated enriched GO terms (e.g. "GP:0005737 cytoplasm" and "Cytoplasm") were manually simplified to keep only one result. Only the top-10 enriched GO terms per species and categories were taken into account, for simplification purposes; all of them with $p$-values $<0.05$.

\section{Declarations}

\section{Ethics approval and consent to participate}

Not applicable.

\section{Consent for publication}

Not applicable.

\section{Availability of data and materials}


The datasets used and/or analysed during the current study are available from the corresponding author on reasonable request.

\section{Competing interests}

The authors declare that they have no competing interests.

\section{Funding}

This work was supported by the Deutsche Forschungsgemeinschaft [AN735/4-1 to M.A.A.N.] and by the H2020-MSCA-RISE project REFRACT - GA No. 823886.

\section{Authors' contributions}

PM conceived and carried out the project. MAAN supervised the project. PM drafted the manuscript. All authors contributed in writing the final manuscript.

\section{Acknowledgements}

Not applicable.

\section{References}

Albà, M.M., Santibáñez-Koref, M.F. \& Hancock, J.M. (2001). The comparative genomics of polyglutamine repeats: extreme differences in the codon organization of repeat-encoding regions between mammals and Drosophila. J. Mol. Evol. 52, 249-259.

Bhattacharyya, A., Thakur, A.K., Chellgren, V.M., Thiagarajan, G., Williams, A.D., Chellgren, B.W. et al. (2006). Oligoproline effects on polyglutamine conformation and aggregation. J. Mol. Biol. 355, 524-535.

Blum, E.S., Schwendeman, A.R. \& Shaham, S. (2013). PolyQ disease: misfiring of a developmental cell death program? Trends Cell Biol. 23, 168-174.

Cuff, J.A. \& Barton, G.J. (1999). Evaluation and improvement of multiple sequence methods for protein secondary structure prediction. Proteins 34, 508-519.

Darnell, G., Orgel J.P.R.O., Pahl, R. \& Meredith, S.C. (2007). Flanking polyproline sequences inhibit betasheet structure in polyglutamine segments by inducing PPII-like helix structure. J. Mol. Biol. 374, 688-704.

Drozdetskiy, A., Cole, C., Procter, J. \& Barton, G.J. (2015). JPred4: a protein secondary structure prediction server. Nucleic Acids Res. 43, W389-W394.

Eftekharzadeh, B., Piai, A., Chiesa, G., Mungianu, D., García, J., Pierattelli, R. et al. (2016). Sequence context influences the structure and aggregation behavior of a polyQ tract. Biophys. J. 110, 2361-2366. 
Escobedo, A., Topal, B., Kunze, M.B.A., Aranda, J., Chiesa, G., Mungianu, D. et al. (2019). Side chain to main chain hydrogen bonds stabilize a polyglutamine helix in a transcription factor. Nat. Commun. 10, 2034.

Faux, N.G., Bottomley, S.P., Lesk, A.M., Irving, J.A., Morrison, J.R., Garcia de la Banda, M., et al. (2005). Functional insights from the distribution and role of homopeptide repeat-containing proteins. Genome Res. 15, 537-551.

Ferrante, M.I., Zullo, A., Barra, A., Bimonte, S., Messaddeq, N., Studer, M. et al. (2006). Oral-facial-digital type I protein is required for primary cilia formation and left-right axis specification. Nat. Genet. $38,112-$ 117.

Guemez-Gamboa, A., Coufal, N.G. \& Gleeson, J.G. (2014). Primary cilia in the developing and mature brain. Neuron 82, 511-521.

Huang, D.W., Sherman, B.T. \& Lempicki, R.A. (2009). Systematic and integrative analysis of large gene lists using DAVID bioinformatics resources. Nat. Protoc. 4, 44-57.

Huang, H., McGarvey, P.B., Suzek, B.E., Mazumder, R., Zhang, J., Chen, Y. et al. (2011). A comprehensive protein-centric ID mapping service for molecular data integration. Bioinformatics 27, 1190-1191.

Jorda, J. \& Kajava, A.V. (2010). Protein homorepeats sequences, structures, evolution, and functions. Adv. Protein Chem. Struct. Biol. 79, 59-88.

Katoh, K. \& Standley, D.M. (2013). MAFFT multiple sequence alignment software version 7: improvements in performance and usability. Mol. Biol. Evol. 30, 772-780.

Lobanov, M.Y. \& Galzitskaya, O.V. (2012). Occurrence of disordered patterns and homorepeats in eukaryotic and bacterial proteomes. Mol. Biosyst. 8, 327-337.

Lobanov, M.Y., Sokolovskiy, I.V. \& Galzitskaya, O.V. (2014). HRaP: database of occurrence of homorepeats and patterns in proteomes. Nucleic Acid Res. 42, D273-D278.

Ludwiczak, J., Winski, A., Szczepaniak, K., Alva, V. \& Dunin-Horkawicz, S. (2019). DeepCoil-a fast and accurate prediction of coiled-coil domains in protein sequences. Bioinformatics 35, 2790-2795.

McMurdie, P.J. \& Holmes, S. (2013). Phyloseq: an R package for reproducible interactive analysis and graphics of microbiome census data. PLoS One 8, e61217.

McMurray, C.T. (2010). Mechanisms of trinucleotide repeat instability during human development. Nat. Rev. Genet. 11, 786-799.

Mier, P., Alanis-Lobato, G. \& Andrade-Navarro, M.A. (2017). Context characterization of amino acid homorepeats using evolution, position, and order. Proteins 85, 709-719. 
Mier, P. \& Andrade-Navarro, M.A. (2018). Glutamine codon usage and polyQ evolution in primates depend on the Q stretch length. Genome Biol. Evol. 10, 816-825.

Mier, P., Pérez-Pulido, A.J. \& Andrade-Navarro, M.A. (2018). Automated selection of homologs to track the evolutionary history of proteins. BMC Bioinformatics 19, 431.

Mier, P., Elena-Real, C., Urbanek, A., Bernadó, P. \& Andrade-Navarro, M.A. (2020). The importance of definitions in the study of polyQ regions: a tale of thresholds, impurities and sequence context. Comput. Struct. Biotechnol. J. https://doi.org/10.1016/j.csbj.2020.01.012. In press.

O’Donoghue, S., Sabir, K.S., Kalemanov, M., Stolte, C., Wellmann, B., Ho, V. et al. (2015). Aquaria: simplifying discovery and insight from protein structures. Nat. Methods 12, 98-99.

Sayers, E.W., Barrett, T., Benson, D.A., Bryant, S.H., Canese, K., Chetvernin, V. et al. (2009). Database resources of the National Center for Biotechnology Information. Nucleic Acids Res. 37, D5-D15.

Schaefer, M.H., Wanker, E.E. \& Andrade-Navarro, M.A. (2012). Evolution and function of CAG/polyglutamine repeats in protein-protein interaction networks. Nucleic Acids Res. 40, 4273-4287.

Sievers, F., Wilm, A., Dineen, D., Gibson, T.J., Karplus, K., Weizhong, L. et al. (2011). Fast, scalable generation of high-quality protein multiple sequence alignments using Clustal Omega. Mol. Syst. Biol. 7, 539 .

Singla, V., Romaguera-Ros, M., Garcia-Verdugo, J.M. \& Reiter, J.F. (2010). Ofd1, a human disease gene, regulates the length and distal structure of centrioles. Dev. Cell 18, 410-424.

Szklarczyk, D., Gable, A.L., Lyon, D., Junge, A., Wyder, S., Huerta-Cepas, J. et al. (2019). STRING v11: protein-protein association networks with increased coverage, supporting functional discovery in genomewide experimental datasets. Nucleic Acids Res. 47, D607-D613.

Thurmond, J., Goodman, J.L., Strelets, V.B., Attrill, H., Gramates, L.S., Marygold, S.J. et al. (2019). FlyBase 2.0: the next generation. Nucleic Acids Res. 47, D759-D765.

Totzeck, F., Andrade-Navarro, M.A. \& Mier, P. (2017). The protein structure context of polyQ regions. PloS One 12, e0170801.

Ward, N. \& Moreno-Hagelsieb, G. (2014). Quickly finding orthologs as reciprocal best hits with BLAT, LAST, and UBLAST: how much do we miss? PLoS One 9, e101850.

Yushchenko, T., Deuerling, E. \& Hauser, K. (2018). Insights into the aggregation mechanism of polyQ proteins with different glutamine repeat lengths. Biophys. J. 114, 1847-1857.

Zerbino, D.R., Achuthan, P., Akanni, W., Amode M.R., Barrel, D., Bhai, J. et al. (2018). Ensembl 2018. Nucleic Acids Res. 46, D754-D761. 


\section{Supplemental Information Note}

\section{Supplementary Files}

Supplementary File 1. Dataset information for the used species. List of the 60 species used in the study, including the dataset name and number of proteins and CDS downloaded from Ensembl.

\section{Figures}

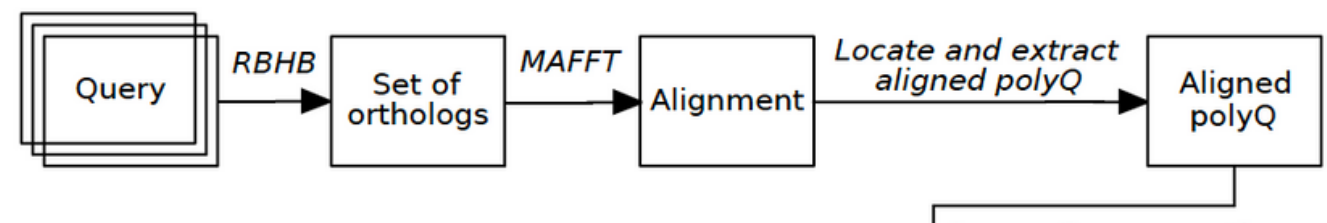

Assess $Q$ conservation per aligned position

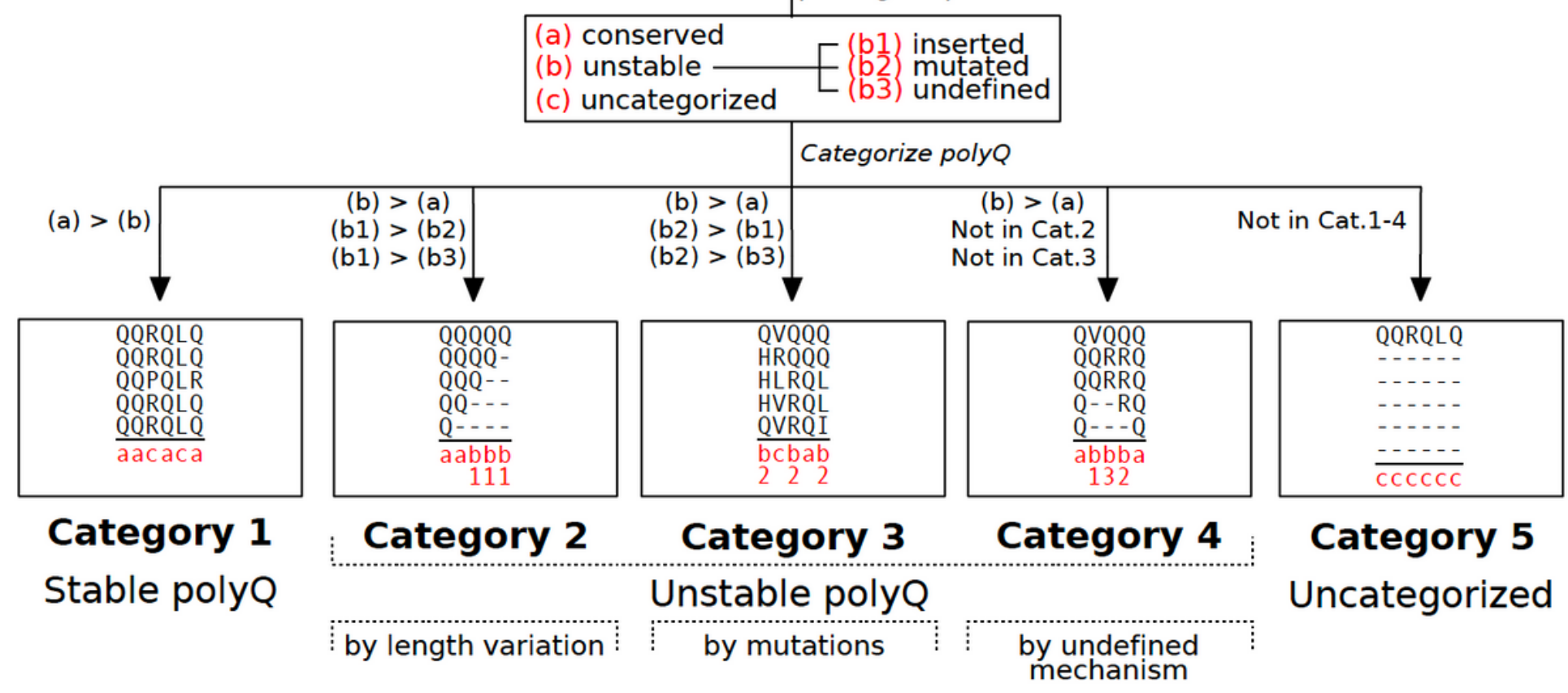

Figure 1

Pipeline for polyQ categorization. Simplification of steps followed to categorize polyQ regions based on their stability when compared to other orthologous regions. RBHB: Reciprocal Best-Hit BLAST. 
a)

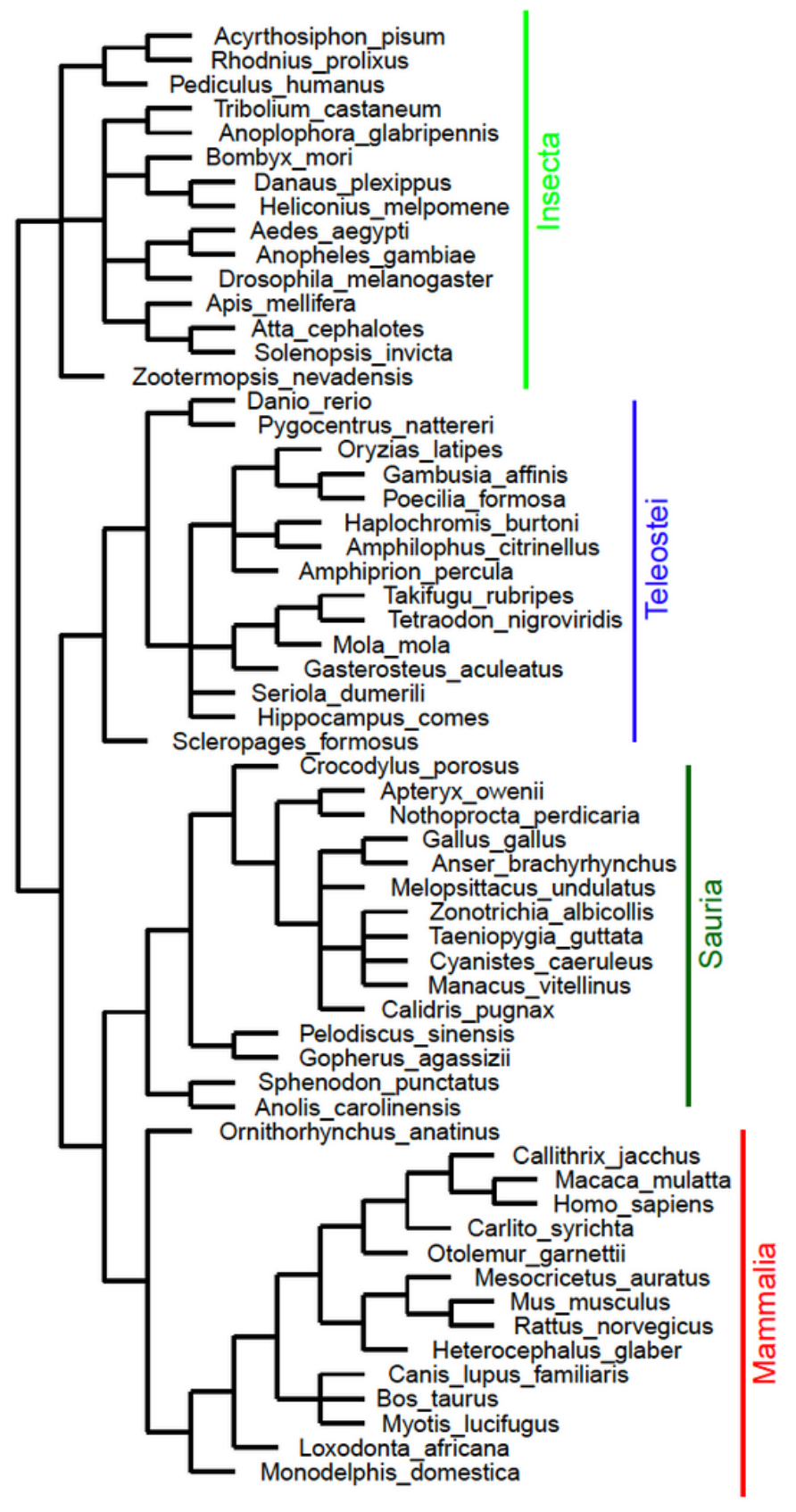

b)

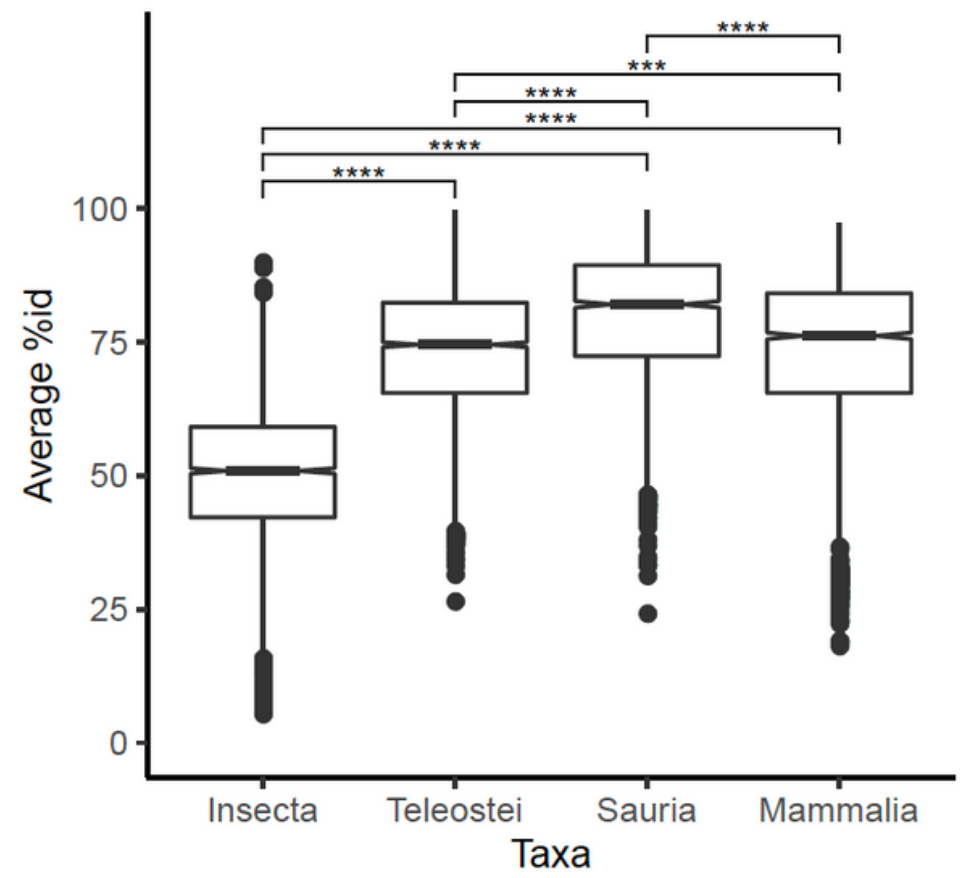

c)

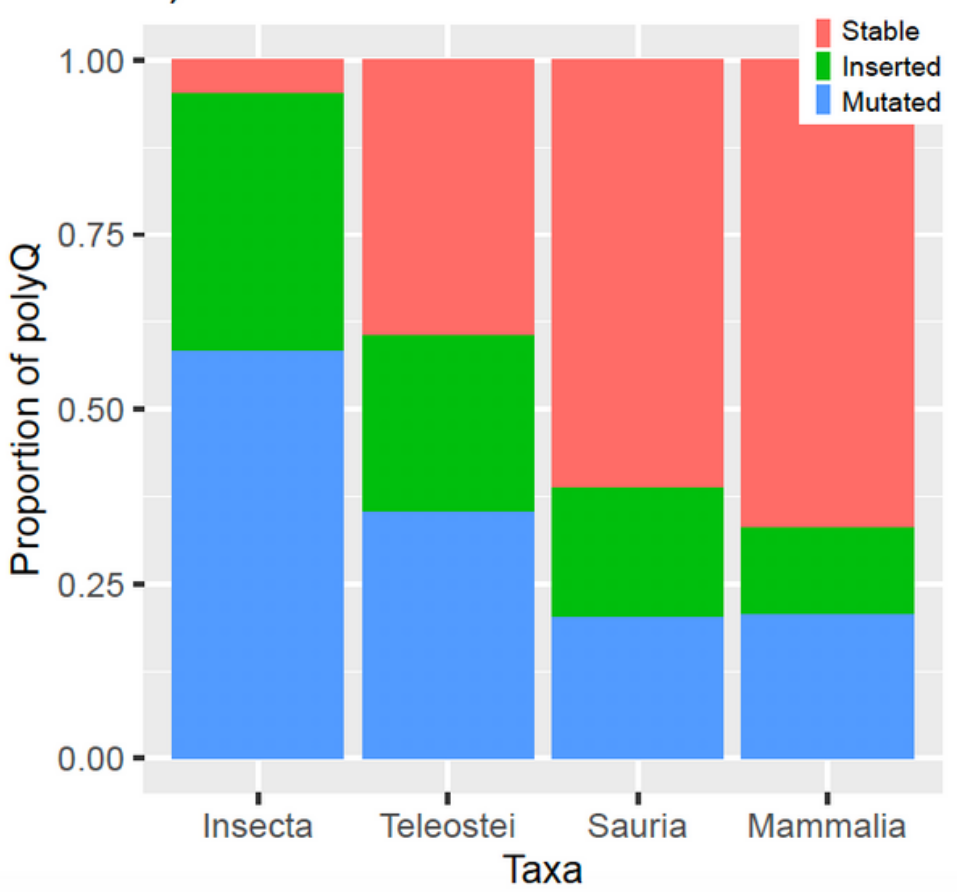

Figure 2

Species selection and categorization of polyQ regions. a) Cladogram of the 60 species used in the present work, 15 per taxon (Insecta, Teleostei, Sauria and Mammalia). b) Average \%identity of proteins in sets of orthologs from different taxa. c) Proportion of polyQ per category per taxa; categories are defined as stable, inserted or mutated polyQ. 

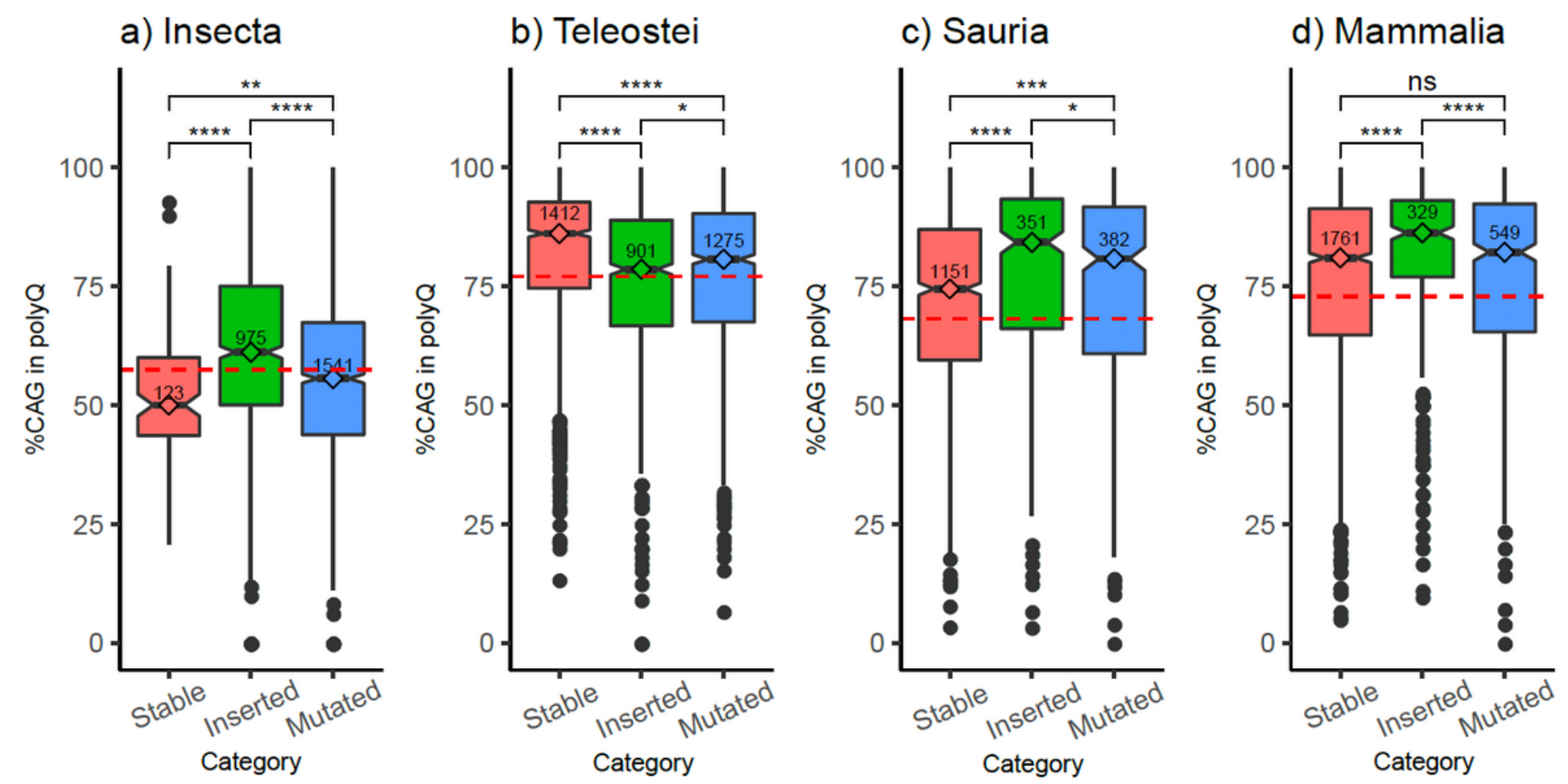

Figure 3

Glutamine codon usage in polyQ regions. \%CAG codons in polyQ regions of orthologous sets of proteins from a) Insecta, b) Teleostei, c) Sauria and d) Mammalia. Categories are defined as: stable polyQ; inserted polyQ; mutated polyQ. Non-parametric Mann-Whitney U statistical tests were performed to compare the distributions ( $* \star \star *$-value $<=1 \mathrm{e}-4$; $* \star \star$ P-value $<=1 \mathrm{e}-3$; $* \star$ P-value $<=0.01$; * P-value $<=0.05$; $\mathrm{ns}=$ not significant). Number of sets of polyQ regions considered per category are shown within each boxplot. 
a)

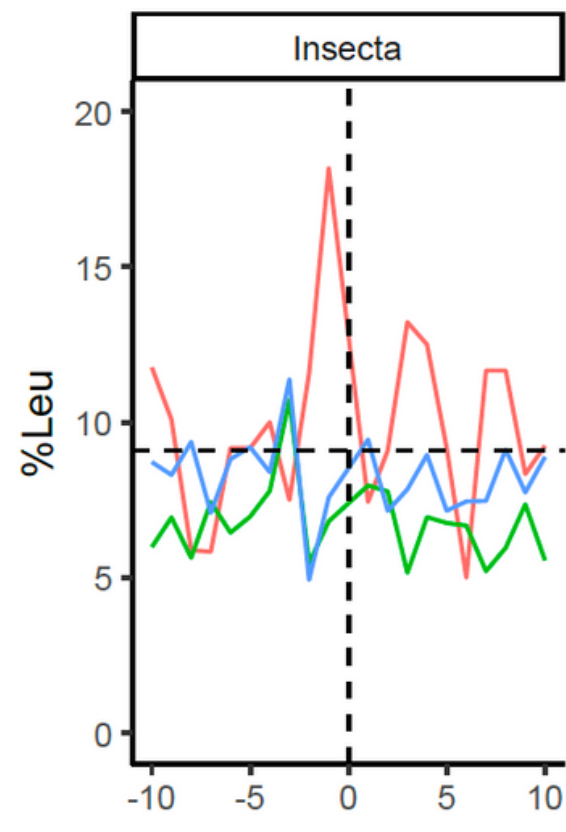

b)
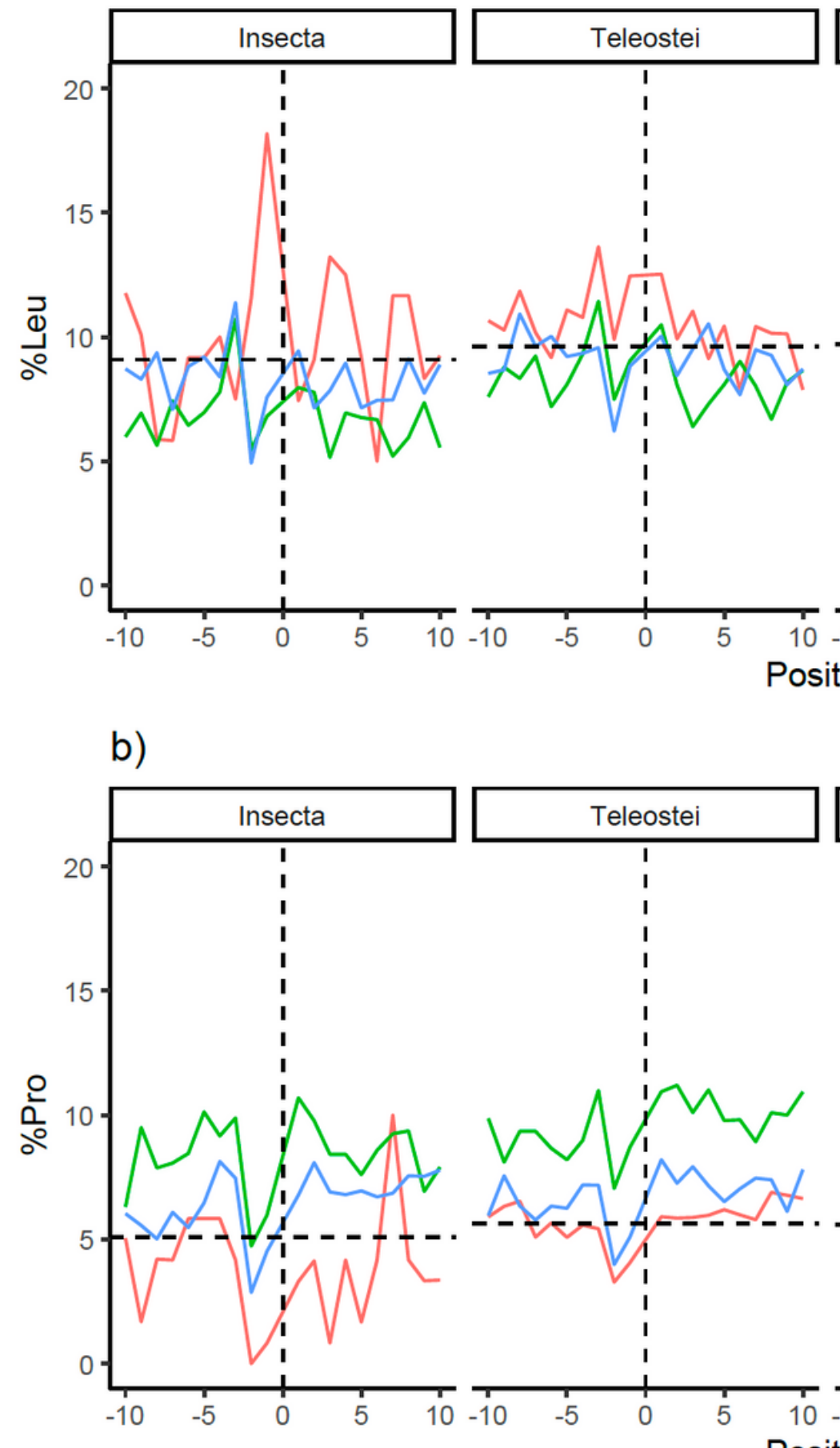

\begin{tabular}{|l}
\hline Stable \\
\hline Sauria \\
\hline
\end{tabular}

Inserted - Mutated
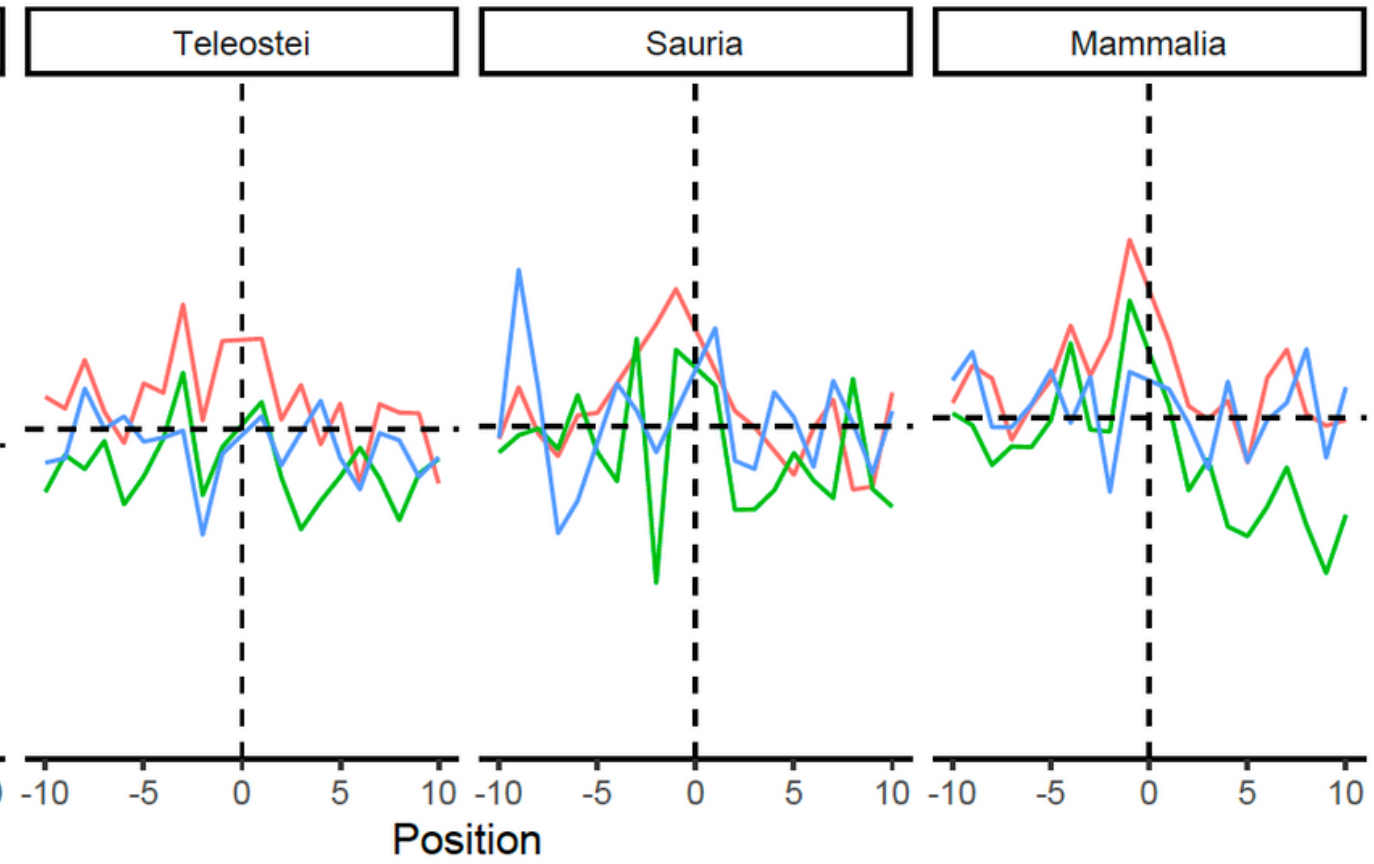

- Stable

- Inserted - Mutated
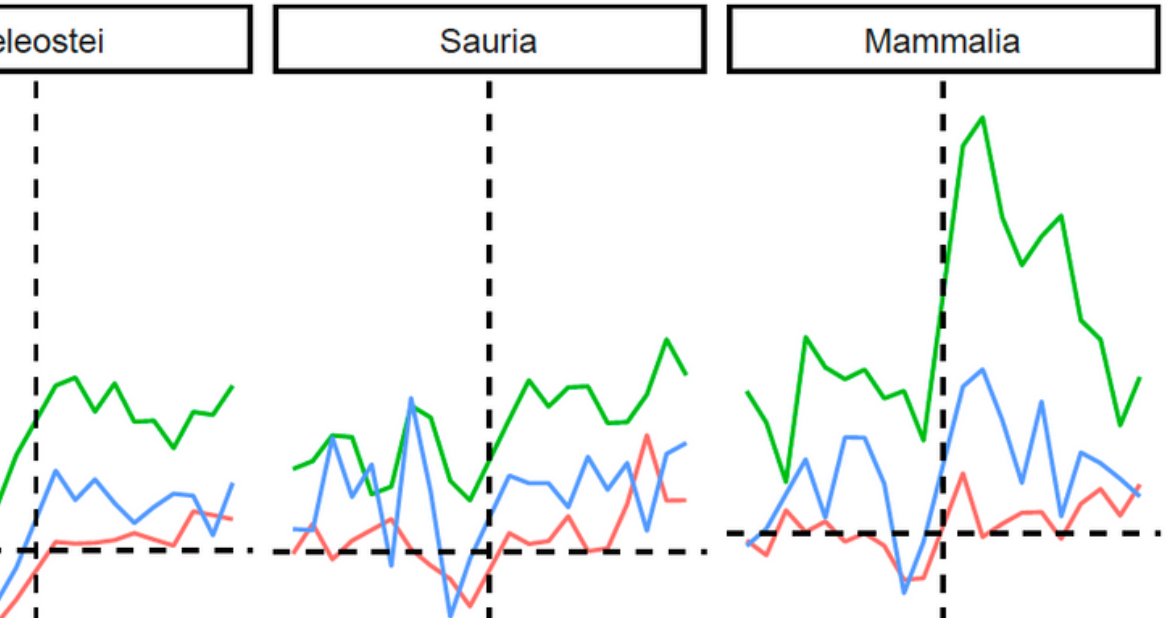

\section{Figure 4}

Amino acid context of polyQ regions. a) Leucine and b) proline abundance around polyQ regions (positions -10 to +10 ) depending on their category and taxa. Vertical lines refer to the position of the polyQ, and horizontal lines to the background composition of the amino acid per taxa. 


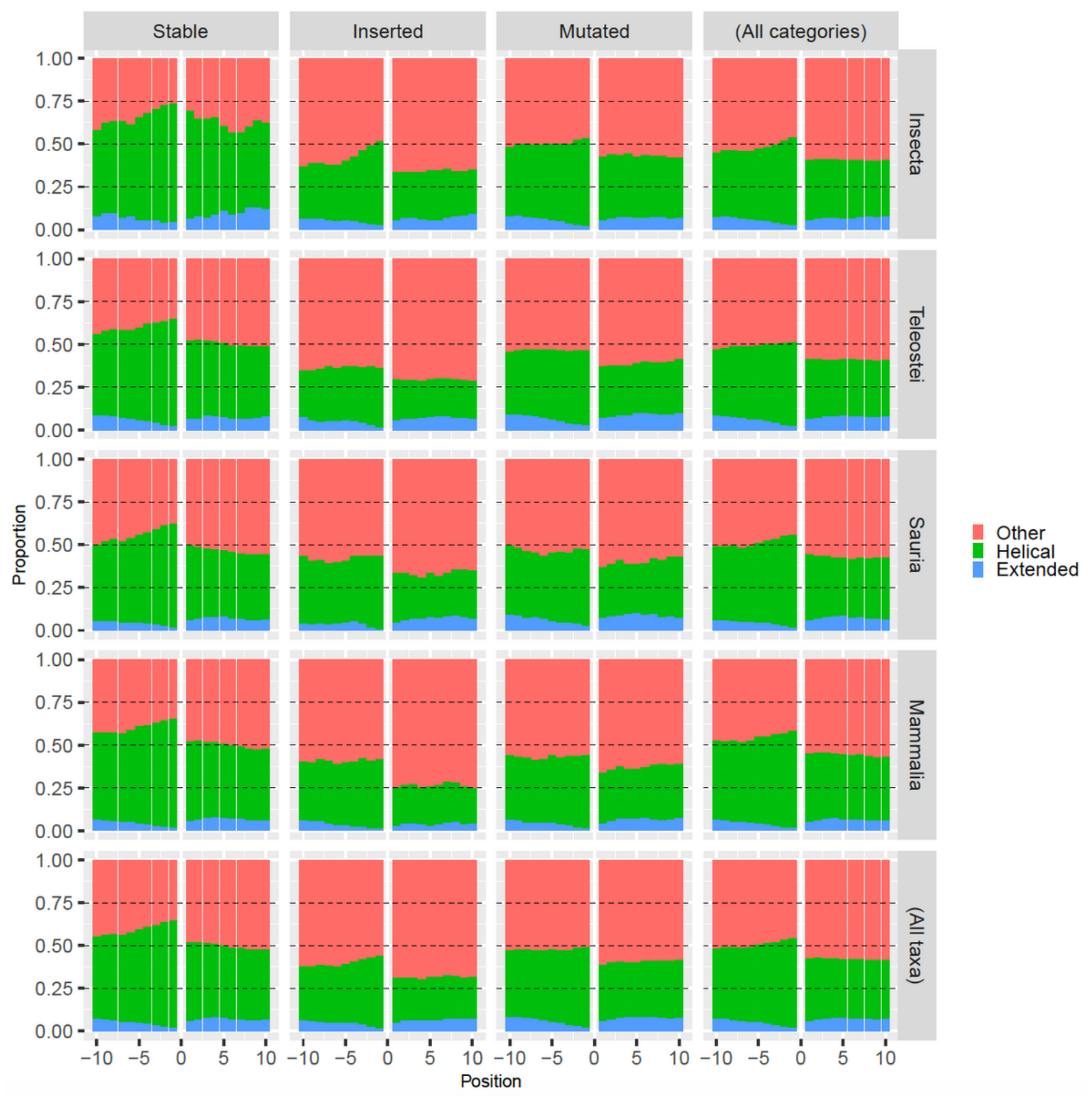

\section{Figure 5}

Secondary structure prediction around polyQ regions. Proportion of predicted alpha helical (green; 'Helical'), beta sheet (blue; 'Extended') and other (red; 'Other') secondary structure conformation in the N(from position -10 to -1 ) and C- (from position +1 to +10 ) terminal regions of polyQ. Results were calculated for proteins in the three polyQ categories and taxa Insecta, Teleostei, Sauria and Mammalia. 
a) Insecta

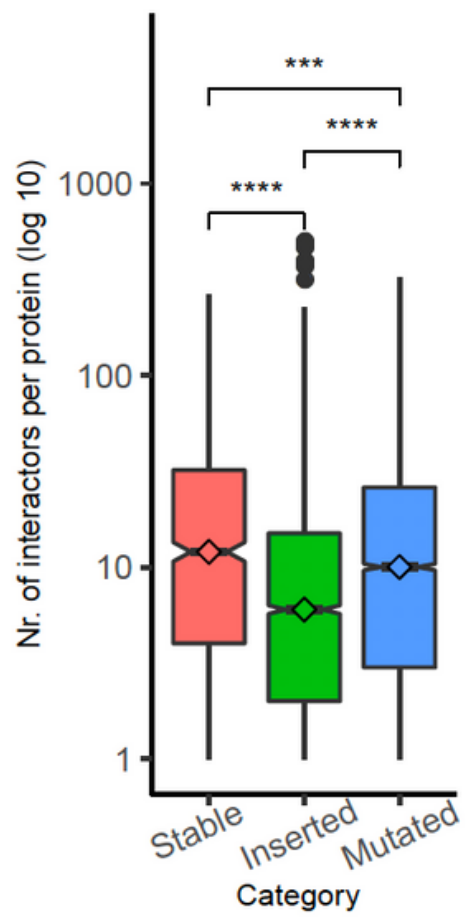

e) Insecta

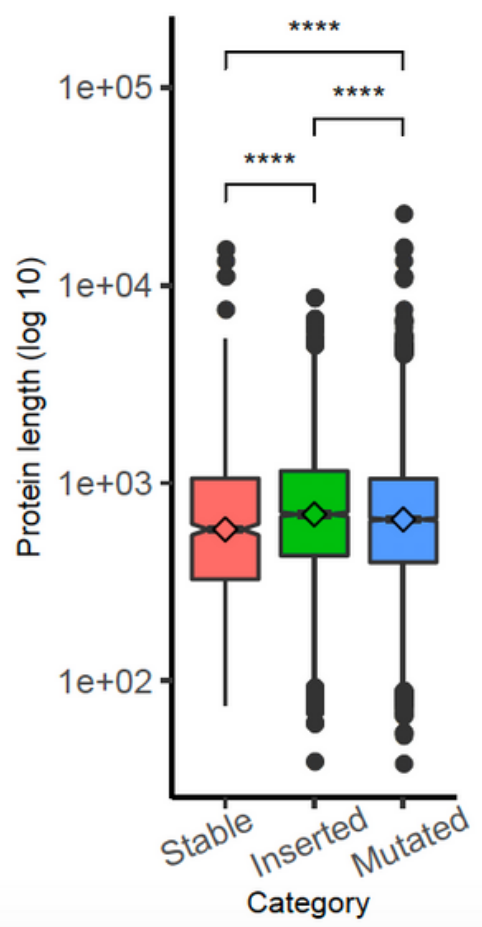

b) Teleostei

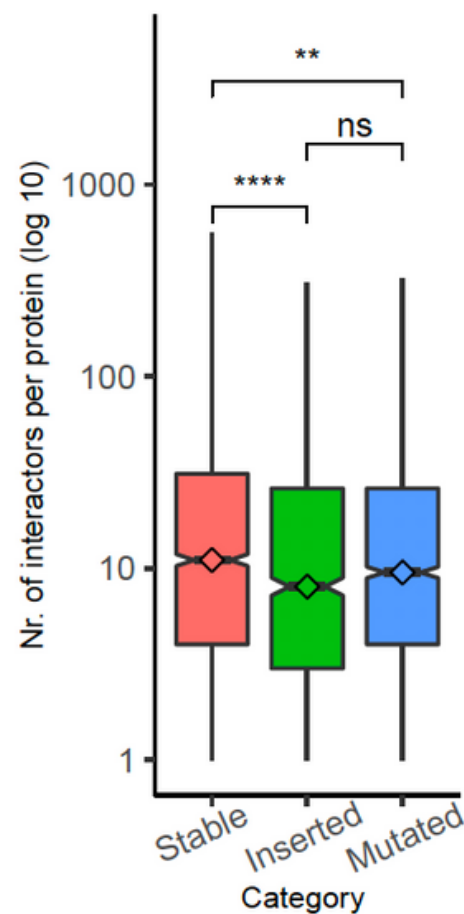

f) Teleostei

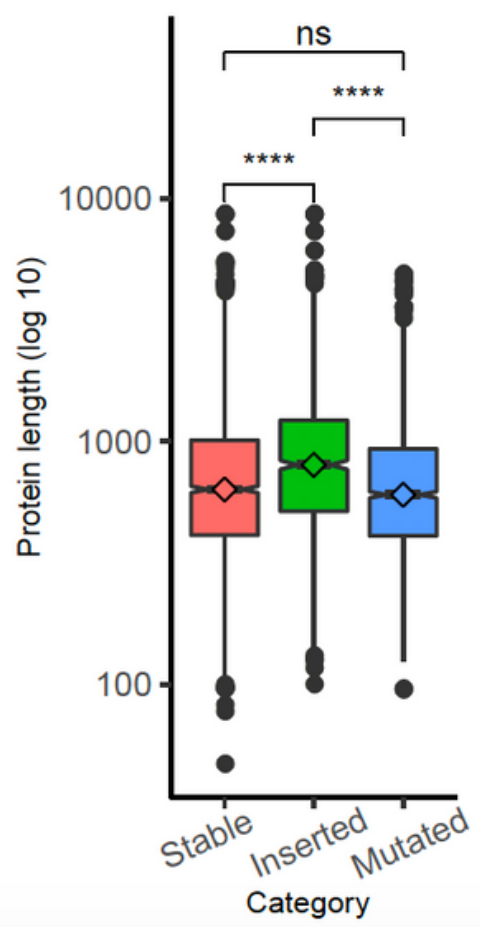

c) Sauria

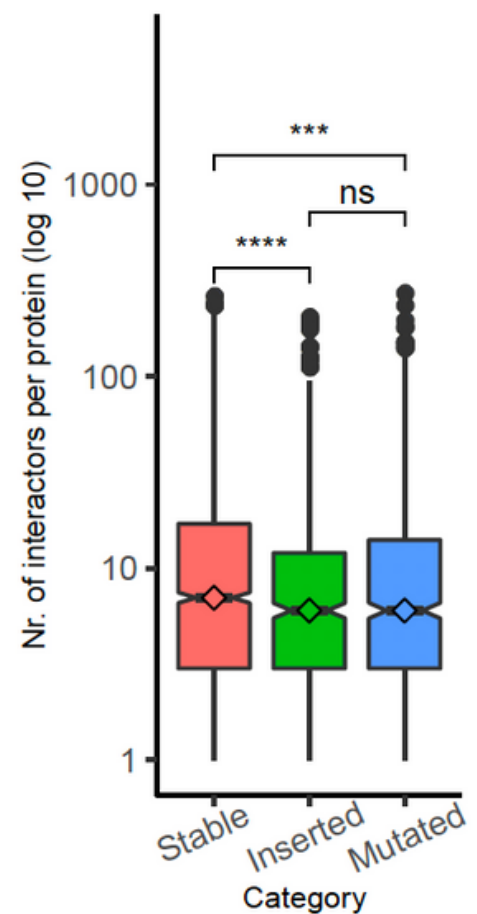

g) Sauria

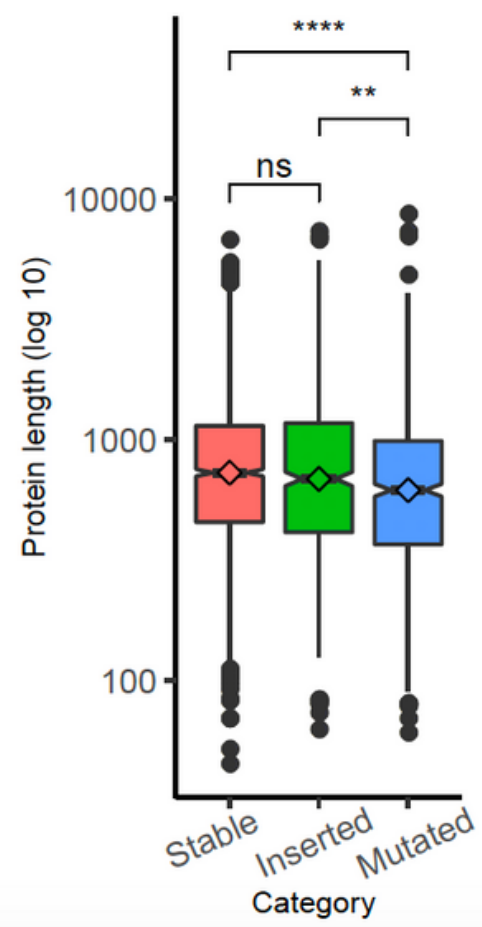

d) Mammalia

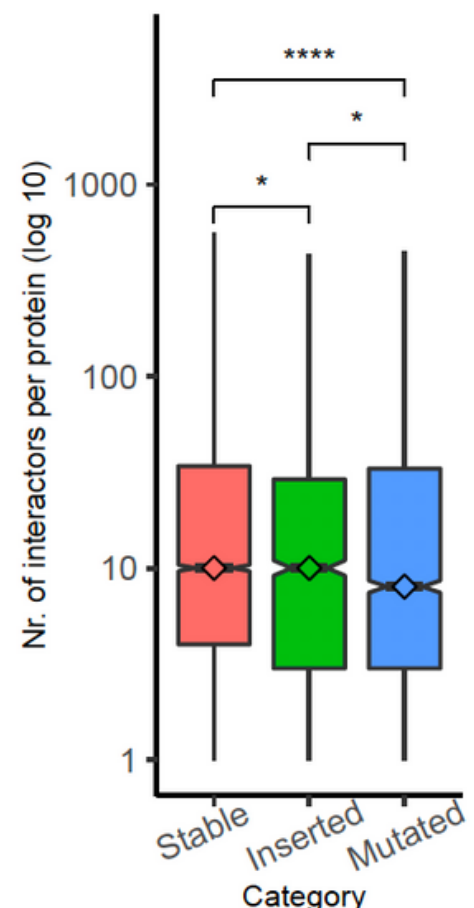

h) Mammalia

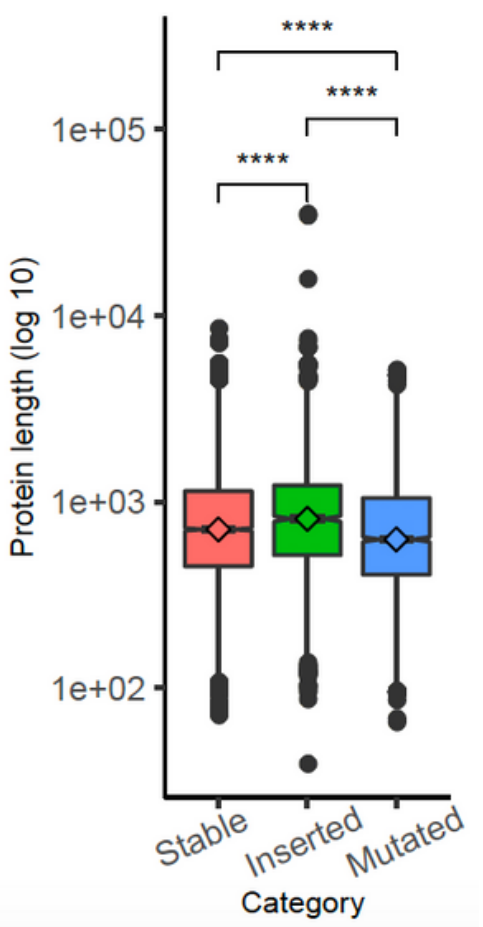

Figure 6

Number of high-confidence interactors per protein. In proteins with at least one interactor. Results are split by polyQ category. Proteins from a) Insecta, b) Teleostei, c) Sauria and d) Mammalia. The length distribution of these proteins are represented for e) Insecta, f) Teleostei, g) Sauria and h) Mammalia. Non-

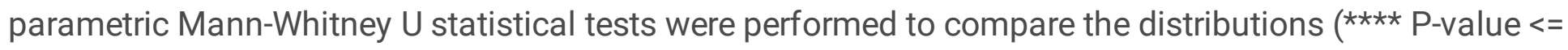

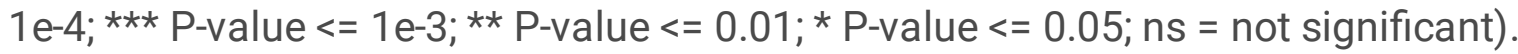




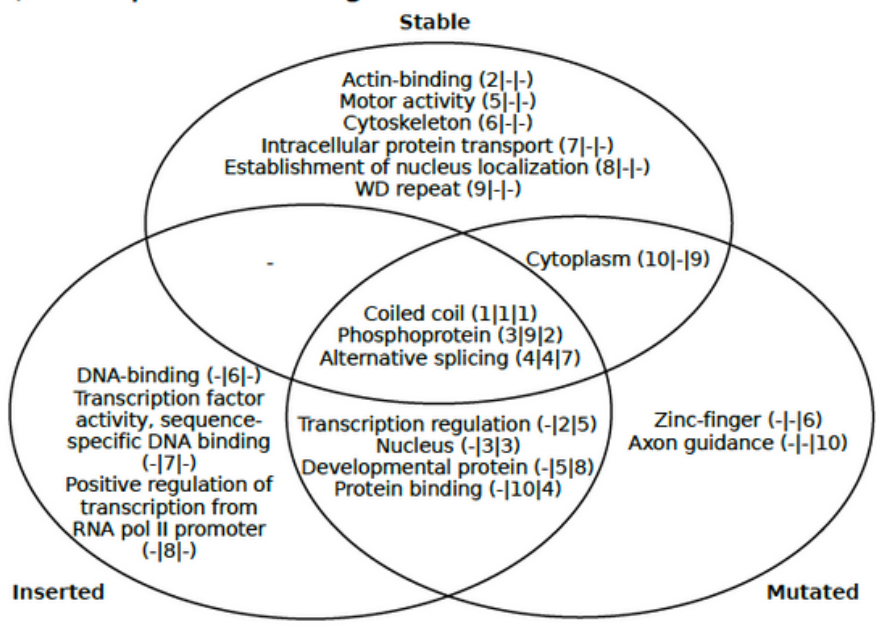

c) Gallus gallus

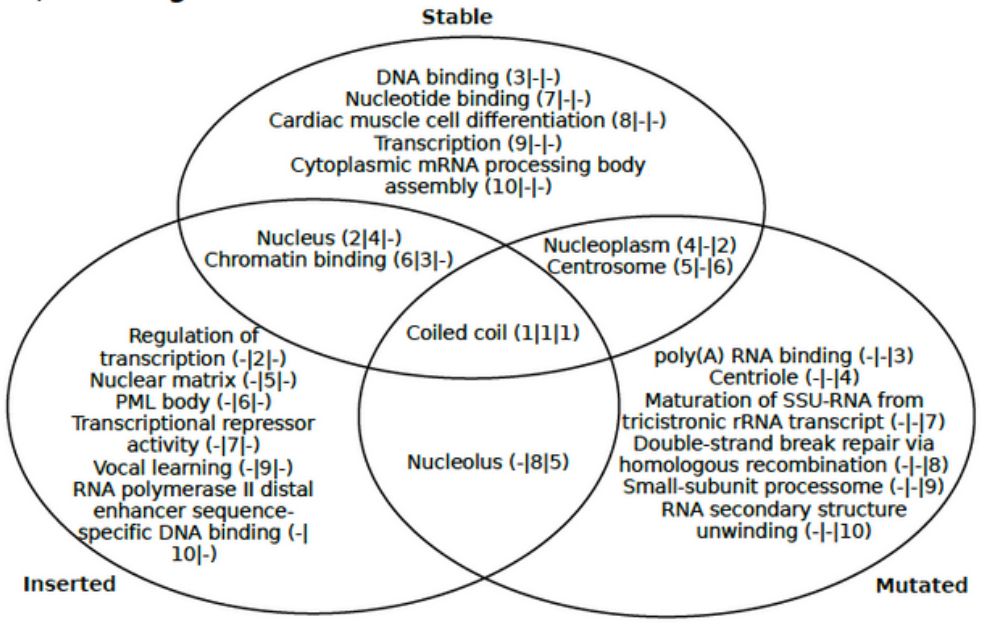

b) Danio rerio

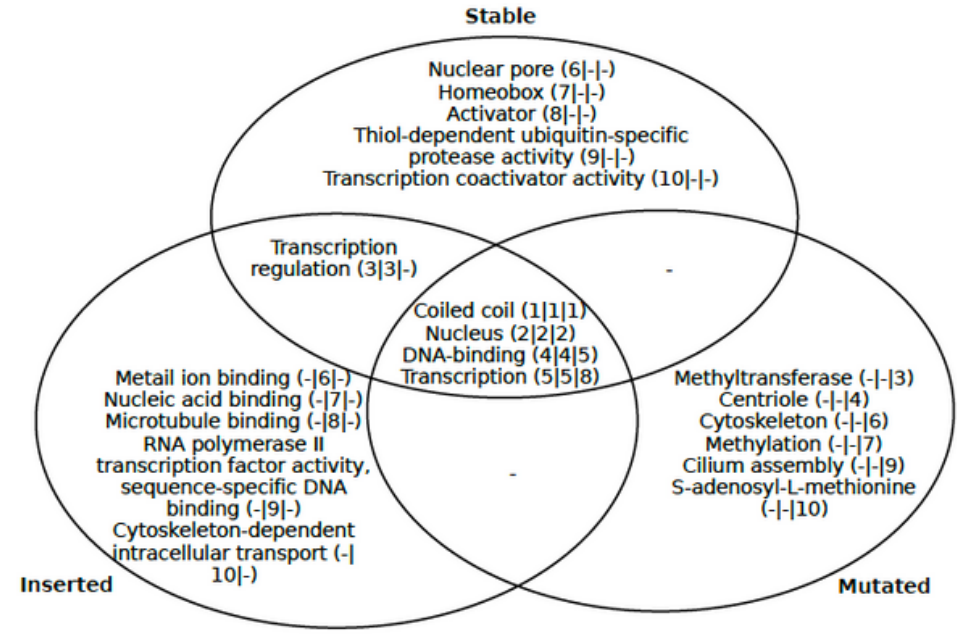

d) Homo sapiens

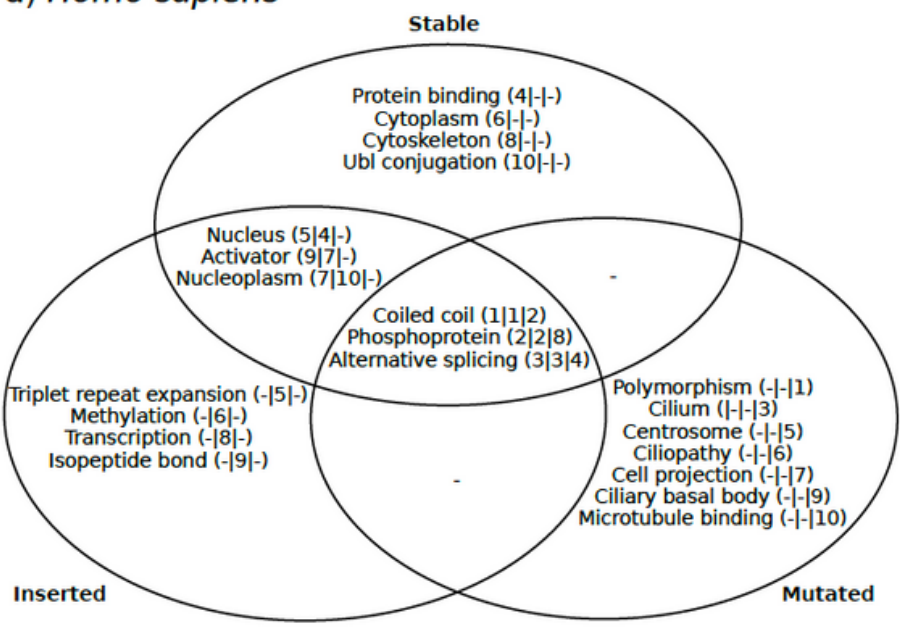

\section{Figure 7}

GO enrichment of proteins in the three polyQ categories. Top-10 enriched GO terms per category in proteins from a) Drosophila melanogaster, b) Danio rerio, c) Gallus gallus and d) Homo sapiens, as representatives of Insecta, Teleostei, Sauria and Mammalia, respectively. Following each Go term we show its position in the top-10 per category in the format '(position in category stable I position in category inserted I position in category mutated)'; a '-' if the term is not present in the top-10 of the category. 
a)

OFD1_HUMAN W5PP93_SHEEP

OFD1_MOUSE D3ZUD5 RAT F7E1B2_ORNAN F1NGR2_CHICK G1K9V8_ANOCA A0A1S3N X89_SALSA H3B0H7_LATCH

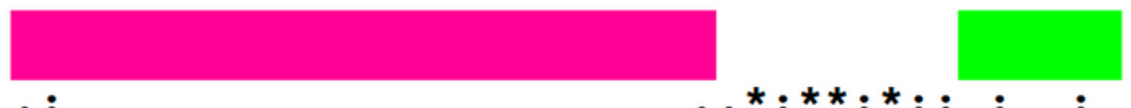

KKELEMENELEMSNQEIKDKSAHSENPLEKYMKI IQQEQDQESADKSSKK EKEYESS - - - VQEMEDKSKSVCGENPLEKYMKIMQQNPDEDTTDKSSKK EEELEKS - - - - VQDQKDKSAHCENTLEKYMKI IQQRQEESNADKSSKK EEELEKS - - - - - VQEQKDKSAHGENPLEKYMKI IQQRQEQSSADKS SKK QKEREGE - - -ELVDITETEGRPAEVNPLEKYMKI IQQSRERGLAEKNSKK EEGDKGEKCESNNSGVEEI IKDSTPNPLEKYMKI IQQRREQELAHEDSTK HEEVT - - ILETSMPQYDKKNGGDI PNPLEKYMKI IQQNREQELVDKNSKE EERGGQEELKEAEEPEEEKAPSSDKDPLQKYLKMIMEQRQSPHVQSPAGR REDASVVKEADASK - DEAVAAGLGASPLEKYMKMIQQHKE - - - QDKNSR

b)

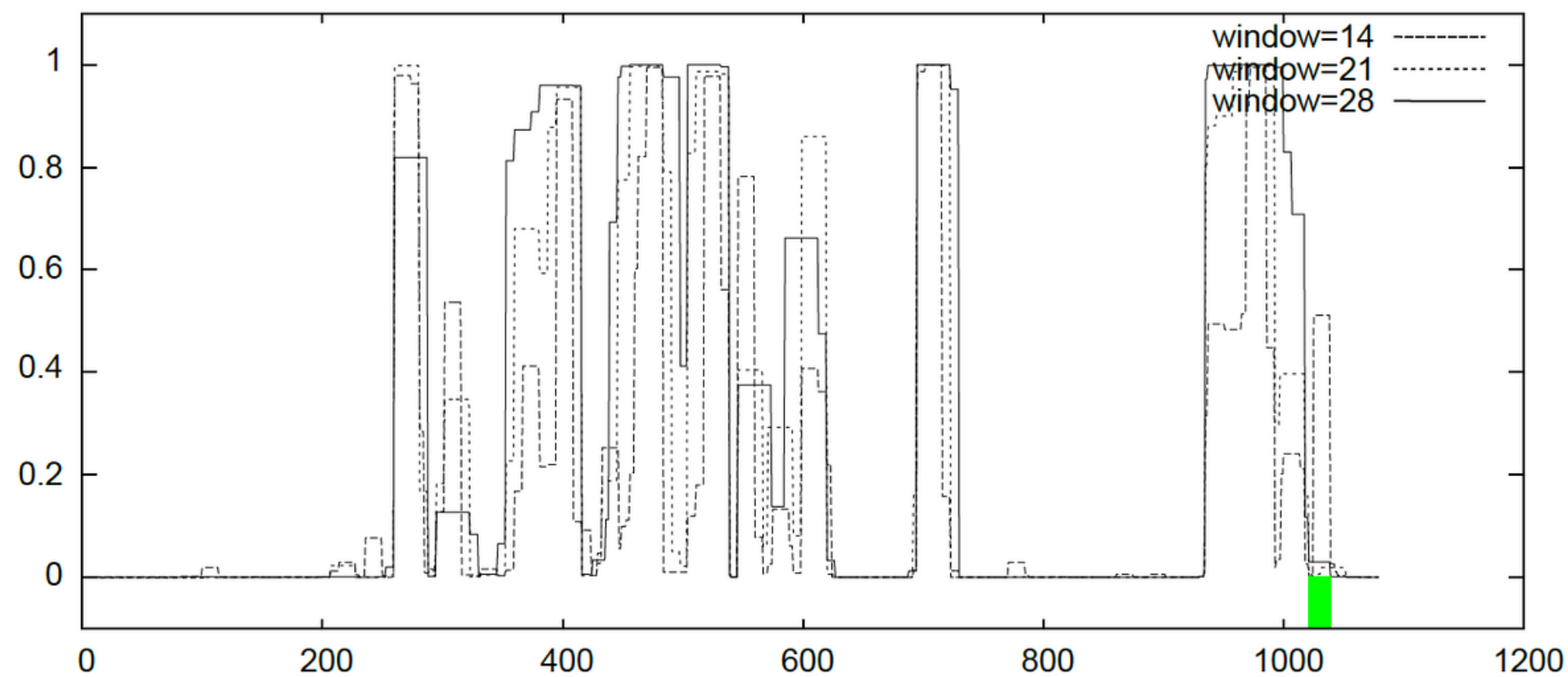

Figure 8

Human OFD1 has a mutated polyQ region at the C-terminal of a predicted coiled coil. a) Part of a multiple sequence alignment of human OFD1 with orthologs in vertebrate species. The end of a predicted coiled coil region (as annotated in the UniProt entry) and the mutated polyQ region are indicated with the magenta and green boxes, respectively. b) Coiled coil prediction from COILS for human OFD1. The position of the polyQ is indicated with a green box.

\section{Supplementary Files}

This is a list of supplementary files associated with this preprint. Click to download.

- SupplFile1.pdf 